\title{
One-dimensional photonic crystal cavities in single-crystal diamond
}

\author{
Luozhou Li ${ }^{\mathrm{a}, 1}$, Tim Schröder ${ }^{\mathrm{a}, 1}$, Edward H. Chen ${ }^{\mathrm{a}, 1}$, Hassaram Bakhru ${ }^{\mathrm{b}}$, \\ Dirk Englund ${ }^{\mathrm{a}}$ \\ ${ }^{a}$ Department of Electrical Engineering and Computer Science, Massachusetts Institute of \\ Technology, Cambridge, MA 02139, USA \\ ${ }^{b}$ College of Nanoscale Science and Engineering, State University of New York \\ Polytechnic Institute, New York, NY 12203, USA
}

\begin{abstract}
The realization of efficient optical interfaces for nitrogen vacancy centers in diamond is an important problem in quantum science with potential applications in quantum communications and quantum information processing. We describe and demonstrate two techniques for fabricating one-dimensional photonic crystal cavities in single-crystal diamond, using (1) a combination of reactive ion etching and focused ion beam milling and (2) transferred silicon hard mask lithography with reactive ion etching. We use two kinds of one-dimensional photonic crystal cavity designs and discuss their optical performances. We find that transferred silicon mask lithography results in better optical properties than focused ion beam patterning techniques. The silicon masks also exhibit high oxygen plasma etching selectivity in excess of 36:1 (diamond:silicon). We use these masks to produce a variety of diamond photonic devices.
\end{abstract}

Keywords:

Quantum information, Photonic crystal, Nitrogen-vacancy center PACS: 42.50.Ct, 42.50.Dv, 42.70.Qs

Email addresses: luozhou@mit.edu (Luozhou Li), englund@mit.edu (Dirk Englund)

${ }^{1}$ These authors contributed equally. 


\section{Introduction}

Solid-state qubits with long spin coherence times are promising for chipintegrated quantum information processing applications. Several different systems for solid-state qubits are currently under investigation [1]. Among them, the negatively charged nitrogen-vacancy (NV) color center in diamond is an exceptional candidate due to its long spin-coherence time even at room temperature [2]. Recent experiments open prospects for use of NV centers in quantum networks [3]. The scalability of the various experimental approaches towards networks requires efficient light-qubit interaction, which calls for the use of cavities.

Coupling the zero-phonon line (ZPL) to an optical cavity mode with a small mode volume $V$ and large quality factor $Q$ strongly enhances the spontaneous emission into the ZPL while suppressing the emission into phonon sidebands, which results in a much higher flux of indistinguishable ZPL photons. Therefore, recently achieved entanglement generation rates of one event per a few minutes between two NV qubits separated by three meters could be enhanced by several orders of magnitude [4]. Such cavity-coupled NV systems would potentially allow for more efficient quantum repeaters [5], quantum microprocessors [6], and quantum networks [7]. Single-shot nondemolition readout of the NV's electronic spin may also be achieved using such cavity-enhanced spin measurements [8].

NV-cavity systems can be realized in both hybrid $[9,10,11,12]$ and allin-diamond $[13,14,15,16]$ approaches. The use of a hybrid system is limited by NV-cavity mode overlap, as well as poor optical and spin properties of nanodiamonds [11]. These drawbacks can be overcome in an all-in-diamond platform, in which the NV would be ideally located at the maximum intensity of the cavity mode in the same diamond. Additionally high-purity single-crystal diamond material allows for high optical and exceptional spin properties. Recent experiments have demonstrated both one-dimensional and two-dimensional photonic crystal (PC) cavities in single-crystal diamond $[13,15,16,17,18,19]$.

Here, we describe and demonstrate two techniques for fabricating onedimensional PC cavities in single-crystal diamond: (1) a combination of reactive ion etching (RIE) and focused ion beam (FIB) milling and (2) transferred silicon hard mask lithography with RIE. 


\section{Experimental}

The RIE-FIB approach described here employs top-down nanofabrication techniques to define large arrays of reactive-ion-etched nanobeams in a single-crystal diamond substrate (nitrogen defect density of 10-1000 parts per billion (ppb)) and then use FIB milling to remove the bottom and suspend the nanobeams (Fig. 1a). Specifically, we used a JBX6300FS electron-beam lithography tool to define the nanobeam patterns in hydrogen silsesquioxane (HSQ). RIE transferred the patterns into bulk diamond with HSQ as a dry etch mask. To determine the quality of vertical etching into the bulk diamond, we coated the HSQ-protected diamond surface with 40-nm-thick $\mathrm{Cr}$ and produced the cross section of one nanobeam with FIB gallium beam milling using a FEI Helios NanoLab 600 dual beam system. The cross section of RIE-etched nanobeams shows straight sidewalls for the first $400 \mathrm{~nm}$ of etching into the diamond (less than $1^{\circ}$ between the sidewalls and the substrate), which is more than enough to cut 200-nm-thick nanobeams (Fig. 1b). The top surface of the diamond nanobeams without cross-section cutting is shown in Fig. 1c(I). To suspend the nanobeams in air, we tilted the sample almost parallel to the gallium beam direction and performed another FIB milling at $30 \mathrm{keV}$ and precision polishing at $2 \mathrm{keV}$ with a gallium ion current of $100 \mathrm{pA}$. After HSQ removal in hydrogen fluoride acid, we noticed that nanobeam gaps were filled with residue as shown in Fig. 1c(II), which was confirmed to contain gallium by energy-dispersive X-ray spectroscopy. Several groups have studied the diffusion of gallium ions implanted by FIB in diamond towards the sample surface and the removal of gallium by annealing in vacuum at temperatures higher than $700^{\circ} \mathrm{C}[20,21,13]$. To remove implanted gallium atoms, we annealed the sample at $1,000^{\circ} \mathrm{C}$ for 2 hours in vacuum. Figure 1c(III) shows the sample after this annealing step. Finally, we attempted to remove FIB-caused graphite layers [22] by oxidation in air $\left(420^{\circ} \mathrm{C}, 6\right.$ hours $)$.

As a second approach, the cavities were also patterned in high-purity single-crystal diamond membranes using a different fabrication process that employs silicon membranes as etch masks [19, 23]. The diamond membrane was fabricated by microwave plasma assisted chemical vapor deposition (CVD), polished to $5-\mu \mathrm{m}$ thickness. NVs were created by implantation of ${ }^{15} \mathrm{~N}$ and subsequent annealing (Fig. 2a). We flipped the diamond membrane and thinned it to $\sim 200 \mathrm{~nm}$ from the backside using chlorine and oxygen reactive ion etching (Fig. 2b). Chlorine plasma etching was performed in 
an Oxford ICP etcher at a flow rate of $40 \mathrm{sccm}$, RIE power of $100 \mathrm{~W}$, and ICP power of $400 \mathrm{~W}$ with an addition of $25 \mathrm{sccm}$ argon. Oxygen plasma etching was performed in Trion RIE at $20 \mathrm{sccm}$ gas flow, 50 mTorr pressure, and $100 \mathrm{~W}$ power. Compared to commercial bulk diamonds with flat and even surfaces, producing uniformly flat diamond membranes with lateral dimensions on the scale of hundreds of microns is more challenging, and the thinned membranes generally exhibited inhomogeneous thicknesses (100 to $300 \mathrm{~nm}$ ) over hundreds of micrometers. To mitigate this problem, we first divided the original membranes into tens of smaller pieces (each approximately $100 \times 100 \mu \mathrm{m}^{2}$ or less in size) and individually thinned the pieces to the targeted thickness. The silicon masks were produced by electron beam lithography and cryogenic plasma etching (sulfur hexafluoride $\left(\mathrm{SF}_{6}\right)$ and oxygen) from silicon-on-insulator wafers with $\sim 220$-nm-thick device layers [24]. These membranes then function as high-quality masks large enough to cover these sub-divided diamond membranes. Specifically, the silicon masks were transferred onto the diamond membranes using a PDMS adhesive (Fig. 2c and $2 \mathrm{~d}$ ). This silicon mask transfer process enables nano-patterning without the need for resist coating on substrates and is compatible with sample sizes as small as several hundreds of square micrometers. We used oxygen plasma [22] to etch the silicon mask pattern into the pre-thinned $\sim 200 \mathrm{~nm}$ diamond membranes (Fig. 2e). An $\mathrm{SF}_{6}$ dry etching removed the bottom silicon and suspended the diamond membrane at device locations (Fig. 2f). By comparing the scanning electron micrographs before and after $\mathrm{SF}_{6}$ etching, we found that $\mathrm{SF}_{6}$ eroded the diamond surface less than $4 \mathrm{~nm}$ during 6 minutes of etch time. The silicon mask can be fabricated with excellent quality, due to the availability of mature fabrication technology for this material. Therefore this process can produce diamond PCs with low surface roughness and uniform, vertical sidewalls.

Then we optically characterized these samples at ambient and cryogenic $(\sim 18 \mathrm{~K})$ temperatures using homebuilt confocal microscope setups with 532$\mathrm{nm}$ continuous-wave laser excitation.

\section{Results and Discussion}

The RIE-FIB method uses RIE for etching diamond nanobeam sidewalls and 'sparingly' applys FIB only to undercut the nanobeam, which reduces the FIB damage compared to all-FIB approaches [25, 26, 27]. We used the cavity design with rectangular air gaps [19]. However, we found that the 
mask patterns produced by electron beam lithography directly on diamond did not produce a high yield of standing structures (15 intact structures in one fabrication run out of 90 written structures), and optical characterization showed no cavity resonances in any of the devices. We attribute the poor results to a combination of reasons. (1) The amorphous layer was not removed completely, which scatters and absorbs light. (2) FIB cutting was not perfectly parallel to the sample surface. Thus the bottom surface of the resultant nanobeams was tilted slightly. (3) The photonic crystals had a small or vanishing band gap overlapping with the cavity frequency due to imperfections on the patterning. Because of this low yield, it was not possible to obtain reliable spectra to evaluate this approach. In addition, CVD grown diamond with a moderate defect density of 10-1000 ppb results in more surface roughness than would be possible with high-purity diamond (defect density $<5 \mathrm{ppb}$ ) [28].

By contrast, we found that cavities produced by transferred silicon mask lithography reliably show optical resonances. We adopted two types of nanobeam cavity designs, one with rectangular air gaps (Figs. 3a, 3b and 3c) and the other with circular air gaps [25] (Figs. 3d and 3e). The optical characterization of nanobeams with rectangular holes is given in Ref.[19], in which we have tuned the cavity peak at low temperature and achieved a Purcell factor enhancement over 60. At the same time, the cavity-coupled NV center shows spin coherence times longer than $200 \mu \mathrm{s}$. Here we focus only on the optical properties of nanobeams with circular holes to complement the work in Ref.[19]. As shown in Fig. 4a, the highest quality factor $(Q)$ we measured is about 1,710 at $568 \mathrm{~nm}$. Fig. 4b shows the spectrum taken at low temperature with a Raman line at $573 \mathrm{~nm}, \mathrm{NV}^{0} \mathrm{ZPL}$ at $575 \mathrm{~nm}, \mathrm{NV}^{-}$ $\mathrm{ZPL}$ at $637 \mathrm{~nm}$, and three cavity resonance peaks at $614 \mathrm{~nm}(Q \sim 860), 688$ $\mathrm{nm}(Q \sim 470)$, and $741 \mathrm{~nm}(Q \sim 570)$. It is possible to tune the $614-\mathrm{nm}$ cavity peak towards the red spectrum via gas deposition [19] and thus enhance $\mathrm{NV}^{-}$ZPL transition rates. We found that the nanobeams with circular holes fabricated here show lower quality factors than in other works $[17,18]$. We determine two effects that degrade the $Q$ factor for circular holes in our experiments: (1) the design is not optimized to tolerate the thickness variation that we find in our membranes, a problem that was addressed in Ref. [16], and (2) the nanobeams with circular holes were arranged too close to each other; in the presented experiments the spacing was about $400 \mathrm{~nm}$, causing optical cross-talk via coupling of the evanescent cavity modes, which degraded $Q$ factors [29]. 
We thus find that between the two approaches described here, transferred silicon mask lithography is advantageous for diamond device fabrication. Using silicon masks, we are able to produce not only high- $Q$ onedimensional PC cavities (highest $Q$ s approaching 10,000) [19], but also high$Q$ two-dimensional PC cavities (highest $Q \mathrm{~s}$ 4,700) [30], while the highest $Q$ reported on FIB-carved diamond cavities is about 700 [13]. Triangular etching using Faraday cages produces high-Q one-dimensional PC cavities as well $[14,18]$, but is probably not compatible with high-Q two-dimensional PC cavities, which require vertically symmetrically patterned high-index layers.

We also found the silicon mask to have higher selectivity in oxygen RIE etching of diamond, compared to an HSQ mask. Specifically, in our experiments we found that 500-nm-thick HSQ etches $2-\mu \mathrm{m}$ diamond without degrading the patterns while 220 -nm silicon mask etches $8-\mu \mathrm{m}$ diamond without degrading. Therefore the etching selectivity of diamond to HSQ is exceeding 4:1 while that of diamond to silicon is exceeding 36:1. As another comparison, we found the selectivity of diamond to SiN to be approximately 8:1 using the same etching recipe. The high aspect ratio that is possible with back-filled silicon masks also serves as a high-selectivity mask for nitrogen ion implantation [31].

\section{Conclusions}

We demonstrate two techniques for fabricating one-dimensional PC cavities in single-crystal diamond: (1) a combination of RIE and FIB, and (2) transferred silicon mask lithography using RIE. The second strategy produces high-quality optical cavities and can be used to fabricate many other different structures into diamond, including circular bullseye gratings [23] and diamond nanowires [32, 33]. Combined with masked implantation [34], the transferred-mask lithography described here serves as a useful tool to pattern microscopic diamond membranes into devices for quantum information processing.

\section{Acknowledgements}

Experiments were supported in part by the Air Force Office of Scientific Research (AFOSR Grant No. FA9550-11-1- 0014, supervised by Gernot Pomrenke). Fabrication was carried out in part at the Center for Functional Nanomaterials, Brookhaven National Laboratory, which is supported by the 
U.S. Department of Energy, Office of Basic Energy Sciences, under Contract No. DE-AC02-98CH10886. T.S. was supported by the Alexander von Humboldt Foundation. E.H.C. was supported by the NASA Office of the Chief Technologist's Space Technology Research Fellowship. We would like to thank Michael Walsh for his help on data analysis.

[1] D. D. Awschalom, L. C. Bassett, A. S. Dzurak, E. L. Hu, J. R. Petta, Quantum spintronics: Engineering and manipulating atomlike spins in semiconductors, Science 339 (6124) (2013) 1174-1179.

[2] M. W. Doherty, N. B. Manson, P. Delaney, F. Jelezko, J. Wrachtrup, L. C. Hollenberg, The nitrogen-vacancy colour centre in diamond, Phys. Rep. 528 (1) (2013) 1-45.

[3] M. Lončar, A. Faraon, Quantum photonic networks in diamond, MRS bulletin 38 (02) (2013) 144-148.

[4] H. Bernien, B. Hensen, W. Pfaff, G. Koolstra, M. Blok, L. Robledo, T. Taminiau, M. Markham, D. Twitchen, L. Childress, , R. Hanson, Heralded entanglement between solid-state qubits separated by three metres, Nature 497 (7447) (2013) 86-90.

[5] L. Childress, J. Taylor, A. S. Sorensen, M. Lukin, Fault-tolerant quantum communication based on solid-state photon emitters, Phys. Rev. Lett. 96 (7) (2006) 070504.

[6] K. Nemoto, M. Trupke, S. J. Devitt, A. M. Stephens, B. Scharfenberger, K. Buczak, T. Nöbauer, M. S. Everitt, J. Schmiedmayer, W. J. Munro, Photonic architecture for scalable quantum information processing in diamond, Phys. Rev. X 4 (3) (2014) 031022.

[7] N. H. Nickerson, J. F. Fitzsimons, S. C. Benjamin, Freely scalable quantum technologies using cells of 5-to-50 qubits with very lossy and noisy photonic links, Phys. Rev. X 4 (4) (2014) 041041.

[8] A. Young, C. Hu, L. Marseglia, J. Harrison, J. O'Brien, J. Rarity, Cavity enhanced spin measurement of the ground state spin of an nv center in diamond, New J. Phys. 11 (1) (2009) 013007.

[9] Y.-S. Park, A. K. Cook, H. Wang, Cavity qed with diamond nanocrystals and silica microspheres, Nano Lett. 6 (9) (2006) 20752079 . 
[10] S. Schietinger, T. Schröder, O. Benson, One-by-one coupling of single defect centers in nanodiamonds to high-q modes of an optical microresonator, Nano Lett. 8 (11) (2008) 3911-3915.

[11] D. Englund, B. Shields, K. Rivoire, F. Hatami, J. Vuckovic, H. Park, M. D. Lukin, Deterministic coupling of a single nitrogen vacancy center to a photonic crystal cavity, Nano Lett. 10 (10) (2010) 3922-3926.

[12] J. Wolters, A. W. Schell, G. Kewes, N. Nusse, M. Schoengen, H. Doscher, T. Hannappel, B. Lochel, M. Barth, O. Benson, Enhancement of the zero phonon line emission from a single nitrogen vacancy center in a nanodiamond via coupling to a photonic crystal cavity, Appl. Phys. Lett. 97 (14) (2010) 141108-141108.

[13] J. Riedrich-Möller, L. Kipfstuhl, C. Hepp, E. Neu, C. Pauly, F. Mücklich, A. Baur, M. Wandt, S. Wolff, M. Fischer, S. Gsell, M. Schreckand, C. Becher, One-and two-dimensional photonic crystal microcavities in single crystal diamond, Nature Nanotech. 7 (2011) 69-74.

[14] M. J. Burek, N. P. de Leon, B. J. Shields, B. J. Hausmann, Y. Chu, Q. Quan, A. S. Zibrov, H. Park, M. D. Lukin, M. Lonc ar, Freestanding mechanical and photonic nanostructures in single-crystal diamond, Nano Lett. 12 (12) (2012) 6084-6089.

[15] A. Faraon, C. Santori, Z. Huang, V. Acosta, R. Beausoleil, Coupling of nitrogen-vacancy centers to photonic crystal cavities in monocrystalline diamond, Phys. Rev. Lett. 109 (3) (2012) 33604.

[16] B. J. Hausmann, B. J. Shields, Q. Quan, Y. Chu, N. P. de Leon, R. Evans, M. J. Burek, A. S. Zibrov, M. Markham, D. J. Twitchen, et al., Coupling of NV centers to photonic crystal nanobeams in diamond, Nano Lett. 13 (12) (2013) 5791-5796.

[17] J. C. Lee, D. O. Bracher, S. Cui, K. Ohno, C. A. McLellan, X. Zhang, P. Andrich, B. Alemán, K. J. Russell, A. P. Magyar, et al., Deterministic coupling of delta-doped nitrogen vacancy centers to a nanobeam photonic crystal cavity, Appl. Phys. Lett. 105 (26) (2014) 261101. 
[18] M. J. Burek, Y. Chu, M. S. Liddy, P. Patel, J. Rochman, S. Meesala, W. Hong, Q. Quan, M. D. Lukin, M. Lončar, High quality-factor optical nanocavities in bulk single-crystal diamond, Nat. Commun. 5 (2014) 5718.

[19] L. Li, T. Schröder, E. H. Chen, M. Walsh, I. Bayn, J. Goldstein, O. Gaathon, M. E. Trusheim, M. Lu, J. Mower, M. Cotlet, M. L. Markham, D. J. Twitchen, D. Englund, Coherent spin control of a nanocavity-enhanced qubit in diamond, Nat. Commun. 6 (2015) 6173 .

[20] J.-i. Fujita, M. Ishida, T. Ichihashi, T. Sakamoto, Y. Ochiai, T. Kaito, S. Matsui, Structure and resonant characteristics of amorphous carbon pillars grown by focused-ion-beam-induced chemical vapor deposition, Jpn. J. Appl. Phys. 41 (2002) 4423.

[21] M. Hamada, T. Teraji, T. Ito, Field-induced effects of implanted ga on high electric field diamond devices fabricated by focused ion beam, Appl. Surf. Sci. 216 (1) (2003) 65-71.

[22] L. Li, M. Trusheim, O. Gaathon, K. Kisslinger, C.-J. Cheng, M. Lu, D. Su, X. Yao, H.-C. Huang, I. Bayn, A. Wolcott, R. M. Osgood, D. Englund, Reactive ion etching: Optimized diamond membrane fabrication for transmission electron microscopy, J. Vac. Sci. Technol. B 31 (2013) 06FF01.

[23] L. Li, E. H. Chen, J. Zheng, S. L. Mouradian, F. Dolde, T. Schröder, S. Karaveli, M. L. Markham, D. J. Twitchen, D. Englund, Efficient photon collection from a nitrogen vacancy center in a circular bullseye grating, Nano Lett. (2015) ASAP.

[24] M. Lipson, Silicon photonics: An exercise in self control, Nature Photon. 1 (1) (2007) 18-19.

[25] T. M. Babinec, J. T. Choy, K. J. Smith, M. Khan, M. Loncar, Design and focused ion beam fabrication of single crystal diamond nanobeam cavities, J. Vac. Sci. Technol. B 29 (2011) 010601.

[26] I. Bayn, B. Meyler, A. Lahav, J. Salzman, R. Kalish, B. A. Fairchild, S. Prawer, M. Barth, O. Benson, T. Wolf, et al., Pro- 
cessing of photonic crystal nanocavity for quantum information in diamond, Diam. Relat. Mater. 20 (2011) 937-943.

[27] I. Bayn, B. Meyler, J. Salzman, R. Kalish, Triangular nanobeam photonic cavities in single-crystal diamond, New J. Phys. 13 (2011) 025018.

[28] I. Bayn, S. Mouradian, L. Li, J. Goldstein, T. Schröder, J. Zhang, E. Chen, O. Gaathon, M. Lu, A. Stein, et al., Fabrication of triangular nanobeam waveguide networks in bulk diamond using singlecrystal silicon hard masks, Appl. Phys. Lett. 105 (2014) 211101.

[29] R. Ohta, Y. Ota, H. Takagi, N. Kumagai, K. Tanabe, S. Ishida, S. Iwamoto, Y. Arakawa, Electro-mechanical q factor control of photonic crystal nanobeam cavity, Jpn. J. Appl. Phys. 52 (4S) (2013) 04CG01.

[30] L. Li, I. Bayn, M. Lu, C.-Y. Nam, T. Schröder, A. Stein, N. C. Harris, D. Englund, Nanofabrication on unconventional substrates using transferred hard masks, Sci. Rep. 5 (2015) 7802.

[31] I. Bayn, E. H. Chen, M. E. Trusheim, L. Li, T. Schröder, O. Gaathon, M. Lu, A. Stein, M. Liu, K. Kisslinger, et al., Generation of ensembles of individually resolvable nitrogen vacancies using nanometer-scale apertures in ultrahigh-aspect ratio planar implantation masks, Nano Lett. (2015) ASAP.

[32] S. L. Mouradian, T. Schröder, C. B. Poitras, L. Li, J. Goldstein, E. H. Chen, J. Cardenas, M. L. Markham, D. J. Twitchen, M. Lipson, et al., The scalable integration of long-lived quantum memories into a photonic circuit, arXiv:1409.7965.

[33] R. Patel, T. Schröder, N. Wan, L. Li, S. Mouradian, E. H. Chen, D. Englund, Efficient photon coupling from a diamond nitrogen vacancy centre by integration with silica fibre, arXiv:1502.07849.

[34] T. Schröder, E. Chen, L. Li, M. Walsh, M. E. Trusheim, I. Bayn, D. Englund, Targeted creation and purcell enhancement of nv centers within photonic crystal cavities in single-crystal diamond, in: CLEO: QELS_Fundamental Science, Optical Society of America, 2014, pp. FW1B-6. 
Figure 1: Cavity fabrication in bulk diamond using RIE-FIB. (a) Illustration of RIE transferring the patterns from HSQ into bulk diamond and FIB cutting the bottom to suspend the nanobeams. (b) The cross section of RIE-etched nanobeams shows straight sidewalls for the first $400 \mathrm{~nm}$ etching into the diamond. The top surface is coated with $\mathrm{Cr}$ to prevent charging during FIB cutting. (c) Scanning electron micrograph (SEM) of a representative nanobeam cavity (I) after RIE, (II) after FIB milling of the bottom diamond, and (III) after annealing at $1,000^{\circ} \mathrm{C}$ for 2 hours in vacuum. (d) SEM of the same nanobeam cavity as in (c) after annealing at $1,000^{\circ} \mathrm{C}$ for 2 hours in vacuum. The sample was tilted by $30^{\circ}$ for imaging.

Figure 2: Illustration of patterning on a diamond membrane using a silicon membrane as an etch mask: (a) NVs were created $\sim 100 \mathrm{~nm}$ below the 5 - $\mu \mathrm{m}$ diamond membrane surface by implantation of ${ }^{15} \mathrm{~N}$ atoms. The diamond was subsequently annealed at $850^{\circ} \mathrm{C}$. (b) The 5 - $\mu \mathrm{m}$ diamond membrane was flipped over on a silicon substrate and thinned by RIE to $\sim 200$-nm thickness. (c) A patterned silicon mask was transferred onto a diamond membrane (less than $300 \mathrm{~nm}$ in thickness, adhering to a bulk silicon substrate) using a micro-PDMS adhesive. (d) The silicon membrane on top of the diamond membrane served as an etch mask for oxygen plasma etching. (e) The diamond membrane was patterned with nanostructures during oxygen etching after subsequent mask removal. (f) An $\mathrm{SF}_{6}$ isotropic dry etching removed the silicon underneath and suspended the diamond membrane at device locations.

Figure 3: SEMs of one-dimensional PC cavities produced by silicon mask method. (a) Side view and (b) top view of an array of one-dimensional PC cavities with rectangular holes. (c) A close-up image of a single one-dimensional PC cavity with rectangular holes. (d) Top view and (e) side view of an array of one-dimensional PC cavities with circular holes.

Figure 4: Optical characterization of one-dimensional PC cavities with circular holes. (a) Measured cavity resonance (black dots) with a quality factor $Q \sim 1,710$ from a Lorentzian fit (blue line). (b) The spectrum taken at low temperature from a different sample with a Raman line at $573 \mathrm{~nm}, \mathrm{NV}^{0} \mathrm{ZPL}$ at $575 \mathrm{~nm}, \mathrm{NV}^{-} \mathrm{ZPL}$ at $637 \mathrm{~nm}$, and three cavity resonance peaks at $614 \mathrm{~nm}, 688 \mathrm{~nm}$, and $741 \mathrm{~nm}$. Inset: normalized second-order autocorrelation measurement with $g^{(2)}(0)=0.378$ for the weakly cavity-coupled NV. 


\section{a}

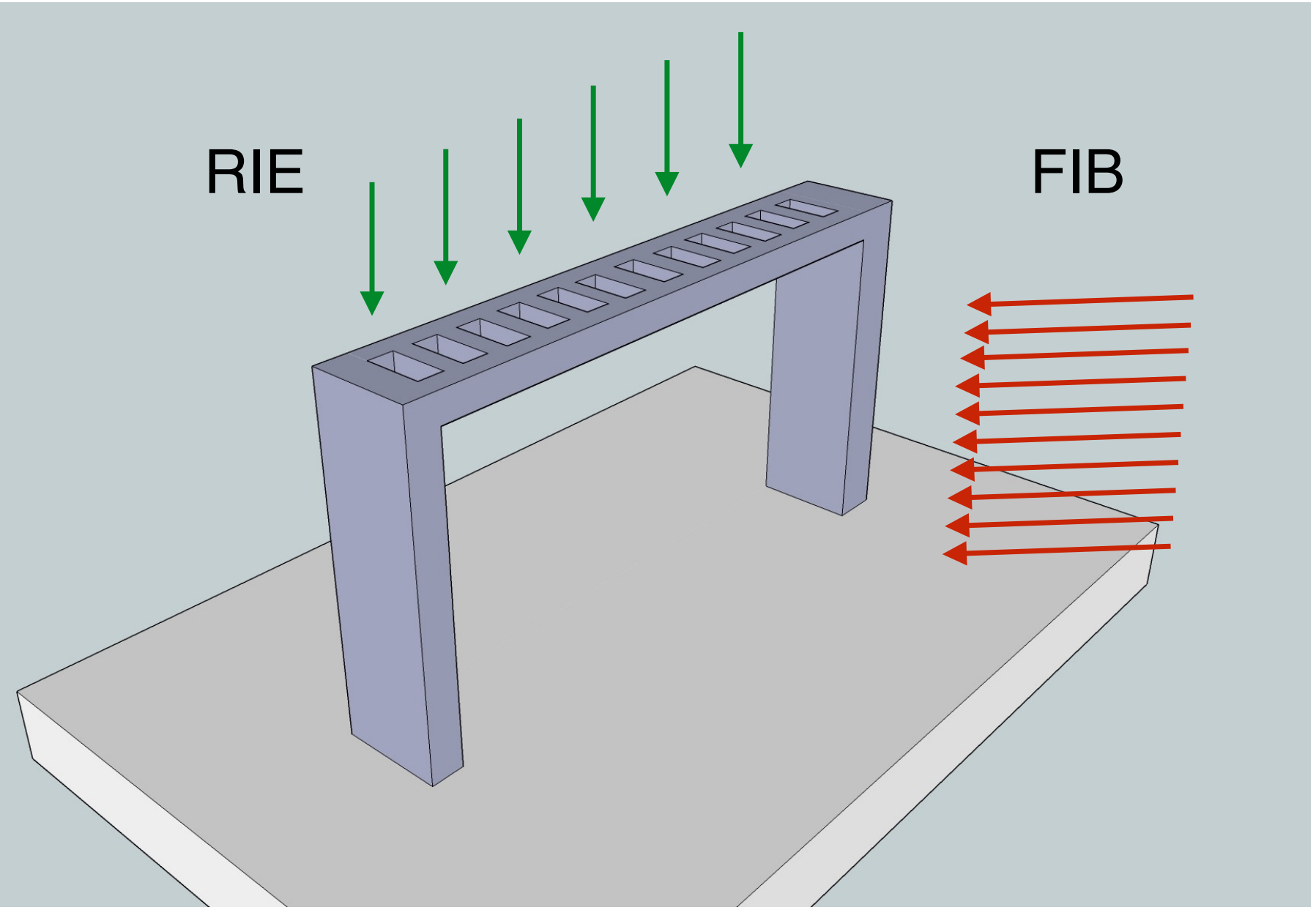

b

HSQ coated with $\mathrm{Cr}$

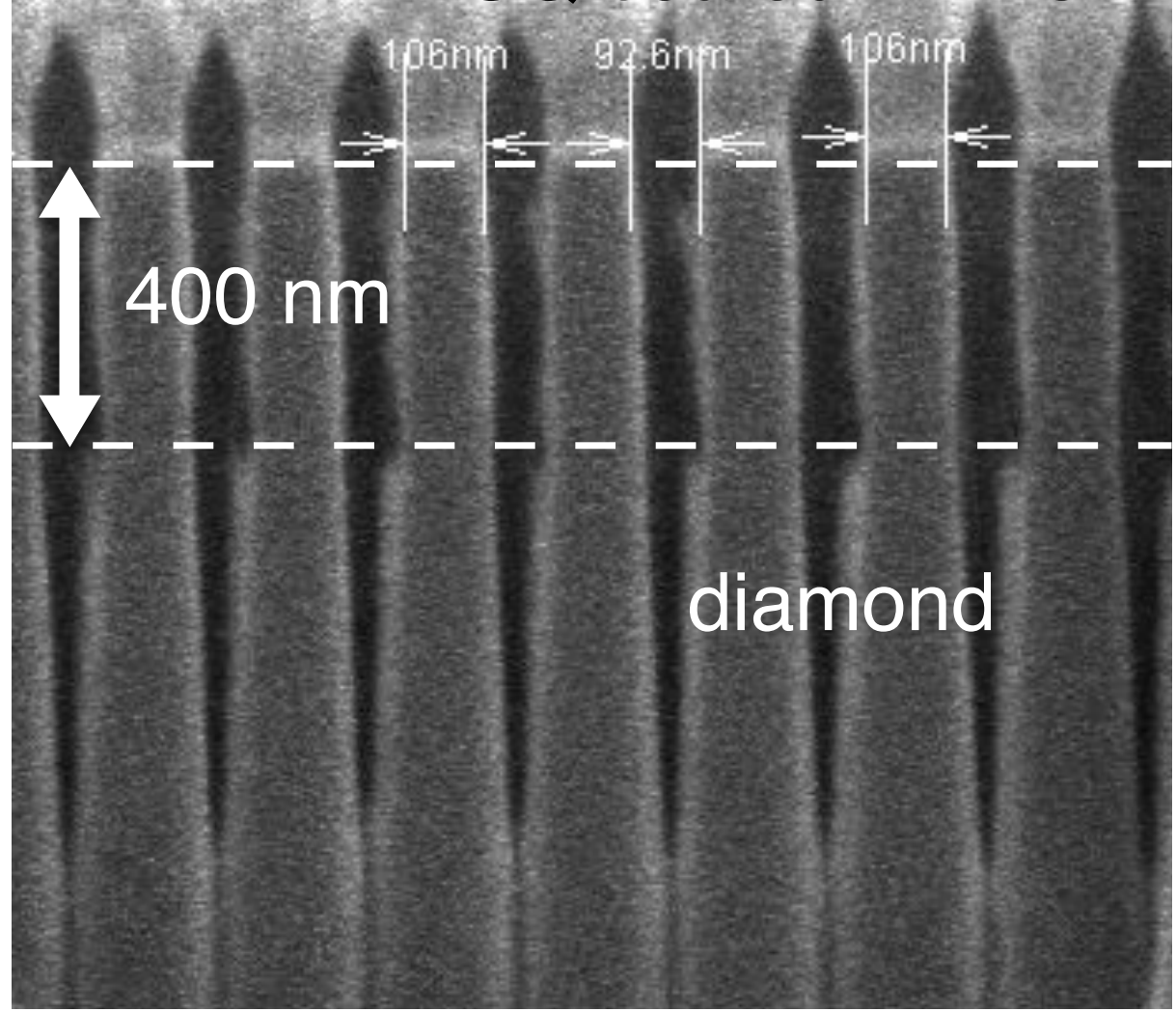

C
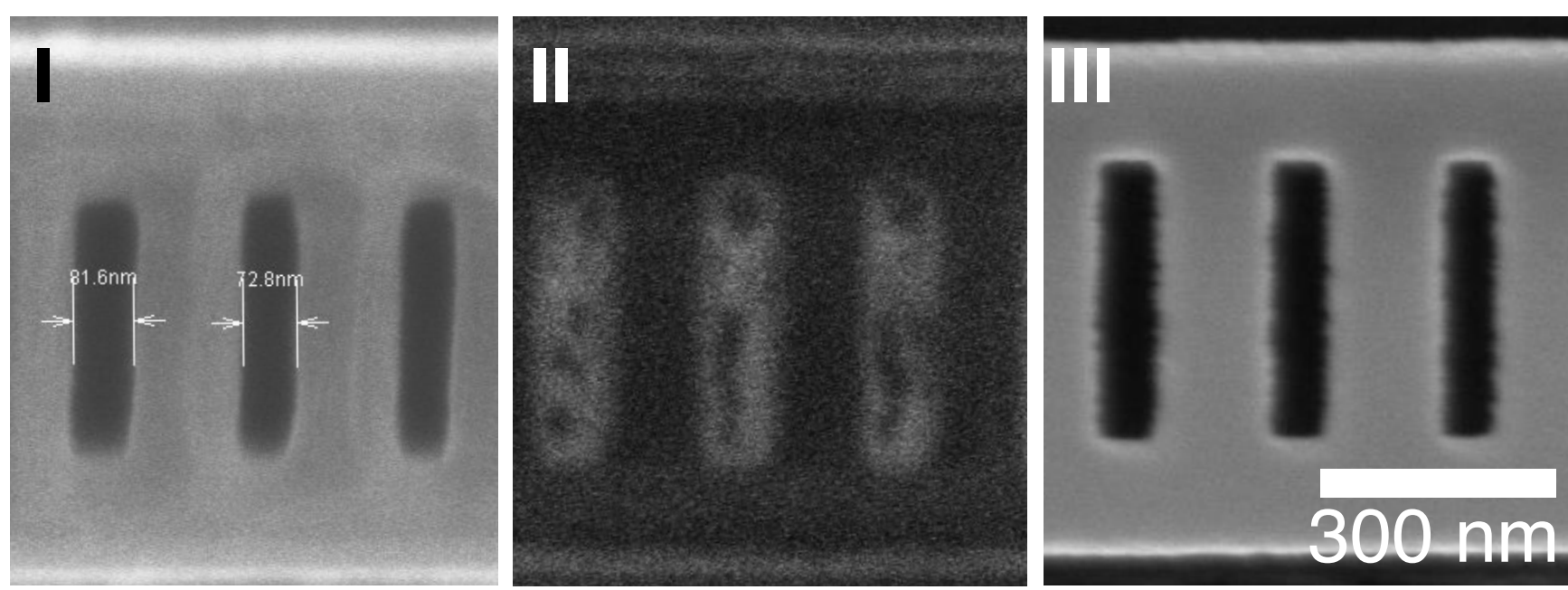

d

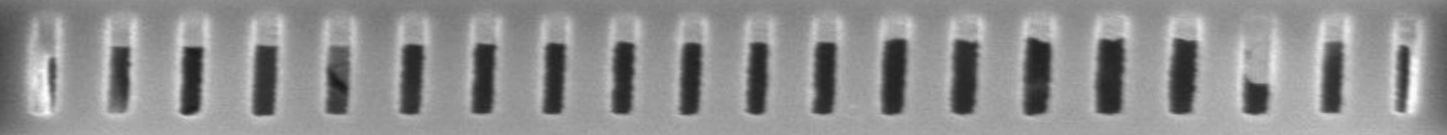

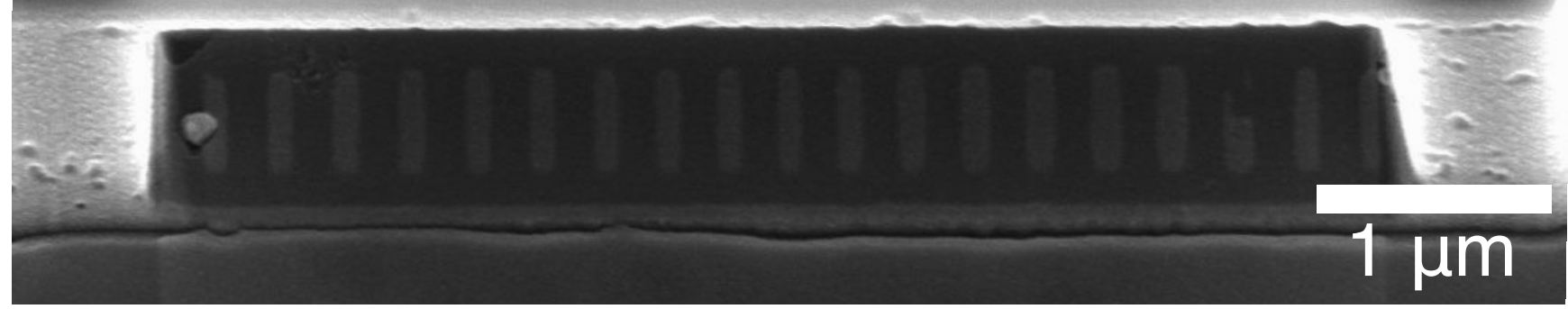


$\mathrm{N}^{+}$implant

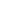




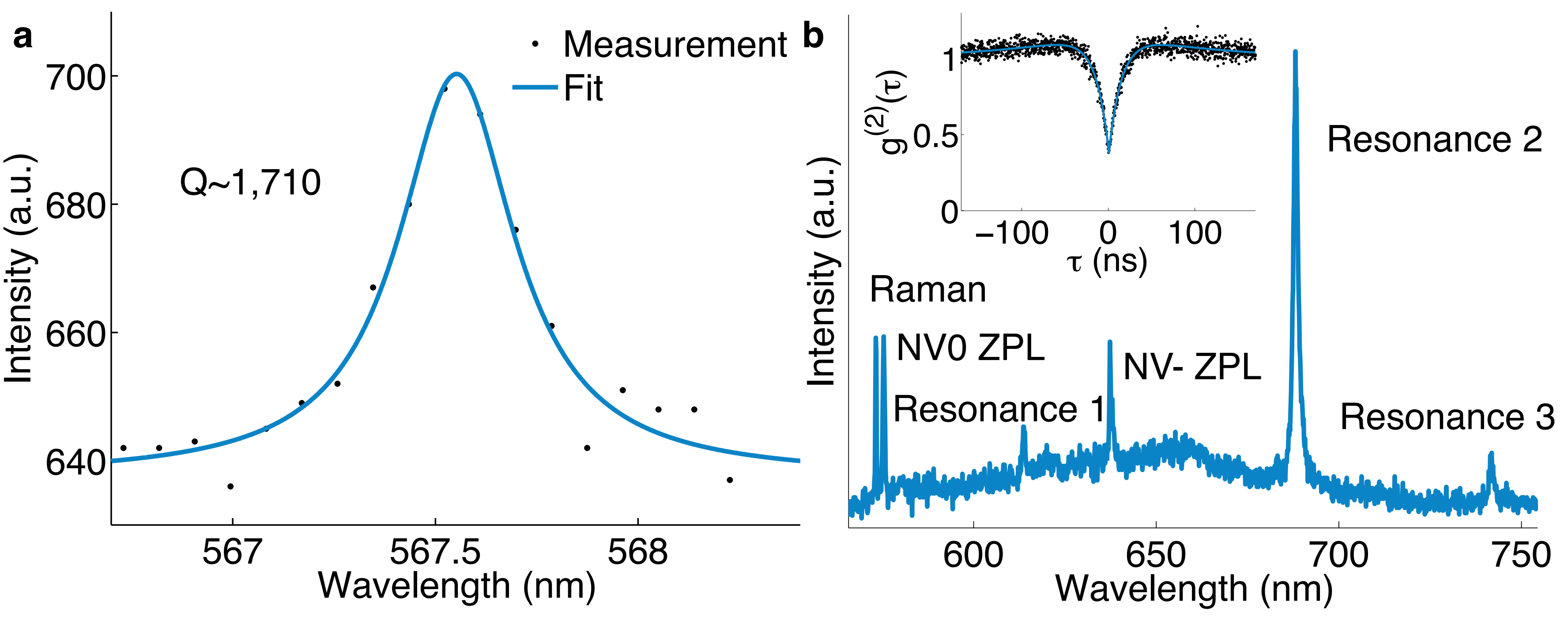

